\title{
NOTE ON MULTIPLICITY
}

\author{
DANIEL KATZ
}

(Communicated by Louis J. Ratliff, Jr.)

\begin{abstract}
Let $(R, M)$ be a local ring with infinite residue field and $I=$ $\left(x_{1}, \ldots, x_{d}\right) R$ an ideal generated by a system of parameters. It is shown that the multiplicity of $I$ equals the multiplicity of $I T$ where

$$
T=\tilde{R}\left[x_{1} / x_{d}, \ldots, x_{d-1} / x_{d}\right]_{M \tilde{R}\left[x_{1} / x_{d}, \ldots, x_{d-1} / x_{d}\right]}
$$

and $\tilde{R}=R /\left(0: x_{d}^{N}\right), N$ large.
\end{abstract}

Introduction. Let $(R, M)$ be a local ring with infinite residue field. A device commonly employed in studying the multiplicity of an $M$-primary ideal is to go mod a superficial element. The effect is to reduce the dimension of the ring yet preserve the multiplicity. This technique is particularly useful in proving theorems about multiplicity by induction on the dimension of $R$. There are however, occasions when this process can be ineffective-certain properties do not lift from homomorphic images. Here we show that this difficulty can sometimes be circumvented by preserving the multiplicity upon passing to subrings of the total quotient ring of $R$ having smaller dimension. To wit, we prove that if $I=\left(x_{1}, \ldots, x_{d}\right) R$ is an ideal generated by a system of parameters, then the multiplicity of $I$ is the same as the multiplicity of $I T$ where

$$
T=\tilde{R}\left[x_{1} / x_{d}, \ldots, x_{d-1} / x_{d}\right]_{M \tilde{R}\left[x_{1} / x_{d}, \ldots, x_{d-1} / x_{d}\right]}
$$

and $\tilde{R}=R /\left(0: x_{d}^{N}\right), N$ sufficiently large. As corollaries we quickly deduce two theorems of D. Rees concerning multiplicity. The first of these, implicit in [5] and proven in [7] under unnecessarily restrictive hypotheses, asserts that if say $R$ is a domain, then there exist finitely many integers $d_{i}>0$ and discrete valuations $v_{i}$ on the quotient field of $R$ such that the multiplicity of $I$ equals $\sum d_{i} v_{i}(I)$. The second is a classical theorem of Rees which guarantees that if $I \subseteq J$ are $M$-primary ideals in a quasi-unmixed local ring then $I$ reduces $J$ if and only if $I$ and $J$ have the same multiplicity. In a brief second section we complete the generalization of a theorem of B. Teissier concerning multiplicities begun by Rees and Sharp in [7].

1. Throughout $(R, M)$ will denote a Noetherian local ring with maximal ideal $M$. We shall assume once and for all that the residue field of $R$ is infinite, though as usual, all of the results below hold without this restriction. For an $M$-primary

Received by the editors January 4, 1988 and, in revised form, March 2, 1988. Presented at the special session in Commutative Algebra at the 841st meeting of the AMS at Knoxville, Tennessee, March 26, 1988.

1980 Mathematics Subject Classification (1985 Revision). Primary 13H15; Secondary 13 C99.

Key words and phrases. Multiplicity, superficial element, integral closure of an ideal.

Partially supported by the General Research Fund at the University of Kansas. 
ideal $I$ and a finitely generated $R$-module $P, e(I, P)$ will denote the multiplicity of $I$ on $P$. That is, if $d=\operatorname{dim}(R)$ then

$$
e(I, P)=\lim _{n \rightarrow \infty} \frac{d !}{n^{d}} l_{R}\left(P / I^{n} P\right)
$$

where $l_{R}(-)$ denotes the length of an $R$-module. In case $R=P$ we set $e(I, R)=$ $e(I)$. We shall make use of two standard properties of $e(I,-)$, namely:

(a) $e(I,-)$ is additive on exact sequences.

(b) If $\operatorname{dim} P<\operatorname{dim} R$ then $e(I, P)=0$.

([2] is a convenient reference for the multiplicity symbol $e(I,-)$.) Recall that an element $g \in I \backslash I^{2}$ is a superficial element for $I$ if there exists a positive integer $c \geq 0$ such that

$$
\left(I^{n}: g\right) \cap I^{c}=I^{n-1}
$$

for $n>c$. Of course, if $I$ is $M$-primary, $e(I)=e(I /(g))$.

THEOREM 1.1. Let $(R, M)$ be a local ring and $I=\left(x_{1}, \ldots, x_{d}\right) R$ an ideal generated by a system of parameters. Set

$$
T=\tilde{R}\left[x_{1} / x_{d}, \ldots, x_{d-1} / x_{d}\right]_{M \tilde{R}\left[x_{1} / x_{d}, \ldots, x_{d-1} / x_{d}\right]}
$$

where $\tilde{R}=R /\left(0: x_{d}^{N}\right), N$ large. Then $e(I)=e(I T)$.

PROOF. We first observe that $e(I)=e(I \tilde{R})$. Apply $e(I,-)$ to the short exact sequence of $R$-modules

$$
0 \rightarrow\left(0: x_{d}^{N}\right) \rightarrow R \rightarrow \tilde{R} \rightarrow 0 .
$$

Then $e(I, R)=e(I, \tilde{R})+e\left(I,\left(0: x_{d}^{N}\right)\right)$. Since $\operatorname{dim}\left(0: x_{d}^{N}\right)<\operatorname{dim} R$ as $R$-modules, $e\left(I,\left(0: x_{d}^{N}\right)\right)=0$. Thus $e(I)=e(I, R)=e(I, \tilde{R})=e(I \tilde{R})$. Consequently, we may replace $\tilde{R}$ by $R$ and write

$$
T=R\left[x_{1} / x_{d}, \ldots, x_{d-1} / x_{d}\right]_{M R\left[x_{1} / x_{d}, \ldots, x_{d-1} / x_{d}\right]} .
$$

The proof now proceeds by induction on $d$, the dimension of $R$. Suppose $d=2$ and $I=\left(x_{1}, x_{2}\right) R$ is generated by a system of parameters. Since $R / M$ is infinite, there exists $y_{1}$ in $R$ satisfying $I=\left(y_{1}, x_{2}\right) R$ and $y_{1}$ is superficial for $I$ (see, for example $[11$, p. 296]).

Let $Z$ be an indeterminate, set $g=x_{2} Z-y_{1}$ and write $S$ for $R(Z)$, the polynomial ring $R[Z]$ localized at $M R[Z]$. We claim that $g$ is superficial for $I S$.

Indeed, suppose $c \geq 0$ satisfies $\left(I^{n}: y_{1}\right) \cap I^{c}=I^{n-1}$ for all $n>c$. Write $f=\sum_{i=0}^{s} r_{i} Z^{i}$, in $R[Z]$ and suppose $f \in\left(I^{n} R[Z]: g\right) \cap I^{c} R[Z]$. Then

$$
f \cdot g=-y_{1} r_{0}+\left(x_{2} r_{0}-y_{1} r_{1}\right) Z+\cdots+\left(x_{2} r_{s-1}-y_{1} r_{s}\right) Z^{s}+x_{2} r_{s} Z^{s+1} .
$$

It follows that $r_{0} \in\left(I^{n}: y_{1}\right) \cap I^{c}$. Thus $r_{0} \in I^{n-1}$, so $x_{2} r_{0} \in I^{n}$. This implies $r_{1} \in\left(I^{n}: y_{1}\right) \cap I^{c}$ so $r_{1} \in I^{n-1}$ Inductively, we conclude that $r_{i} \in I^{n-1}$ for $0 \leq i \leq s$, so $f \in I^{n-1} R[Z]$.

Thus, $g$ is a superficial element for $I R[Z]$ and it remains such for $I R(Z)$ since $I^{n} R(Z) \cap R[Z]=I^{n} R[Z]$ for all $n$. Note that $I S=\left(g, x_{2}\right) S$ and $e(I)=e(I S)=$ $e(I S /(g))$. 
Now let $K$ be the kernel of the natural homomorphism from $S$ to $T$ which sends $Z$ to $y_{1} / x_{2}$ and set $L=(g) S$. We argue as above to show $e(I S / L)=e(I T)$. View

$$
0 \rightarrow K / L \rightarrow S / L \rightarrow T \rightarrow 0
$$

as an exact sequence of $S / L$-modules and consider $\tilde{e}(-) \equiv e(I S / L,-)$, the multiplicity of $I S / L$ on each of these. Then $\tilde{e}(S / L)=\tilde{e}(T)+\tilde{e}(K / L)$. Since $x_{2}^{N} K \subseteq L$ for $N$ large, $\operatorname{dim} K / L<\operatorname{dim} S / L$ (as $S / L$-modules) so $\tilde{e}(K / L)=0$. Thus

$$
e(I)=e(I S)=e(I S / L)=\tilde{e}(S / L)=\tilde{e}(T)=e(I T)
$$

as desired.

Now suppose the result holds for local rings of dimension less than $d$. As before, we may select $y_{1}$ in $R$ such that $I=\left(y_{1}, x_{2}, \ldots, x_{d}\right) R$ and $y_{1}$ is superficial for $I$. Then exactly the same proof shows that $e(I)=e\left(I T_{1}\right)$ where

$$
T_{1}=R\left[y_{1} / x_{d}\right]_{M R\left[y_{1} / x_{d}\right]} \text {. }
$$

Since $\left(T_{1}, M T_{1}\right)$ is $d-1$ dimensional and $I T_{1}=\left(x_{2}, \ldots, x_{d}\right) T_{1}$ is generated by a system of parameters, by induction we have that $e\left(I T_{1}\right)=e\left(I T^{*}\right)$ where

$$
T^{*}=T_{1}\left[x_{2} / x_{d}, \ldots, x_{d-1} / x_{d}\right]_{M T_{1} R\left[x_{2} / x_{d}, \ldots, x_{d-1} / x_{d}\right]} .
$$

However, it is readily seen that

$$
T^{*}=R\left[y_{1} / x_{d}, \ldots, x_{d-1} / x_{d}\right]_{M R\left[y_{1} / x_{d}, \ldots, x_{d-1} / x_{d}\right]}=T .
$$

Thus $e(I)=e\left(I T_{1}\right)=e\left(I T^{*}\right)=e(I T)$ and the proof is complete.

Corollary 1.2 (see [5, Theorem 2.3 and 7 , Theorem 4.3]). Let $(R, M)$ be an analytically unramified local domain and $J \subseteq R$ an $M$-primary ideal. There exist finitely many integers $d_{i}>0$ and discrete valuations $v_{i}$ on the quotient field of $R$ such that $e(J)=\sum d_{i} v_{i}(J)$.

PROOF. Since $R / M$ is infinite, there exists an ideal $I=\left(x_{1}, \ldots, x_{d}\right) R$ generated by a system of parameters such that $I \subseteq J$ and $I J^{n}=J^{n+1}$ for $n$ large (see [3]). Consequently $e(I)=e(J)$. Let $T$ be as in the theorem so $e(J)=e(I)=e(I T)$. Since $R$ is analytically unramified, $T^{\prime}$, the integral closure of $T$, is a finitely generated $T$-module [4]. If we write $M_{1}, \ldots, M_{t}$ for the maximal ideals of $T^{\prime}$ then

$$
e(I T)=\sum_{i}\left[T^{\prime} / M_{i}: T / M T\right] e\left(I T_{M_{i}}^{\prime}\right)
$$

where $\left[T^{\prime} / M_{i}: T / M T\right]$ is the algebraic degree of the extension $T^{\prime} / M_{i} \supseteq T / M T$ ([11], p. 299). Since $T_{M_{i}}^{\prime}$ is a $D V R$ with associated valuation $v_{i}, e\left(I T_{M_{i}}^{\prime}\right)=v_{i}(I)$. The relation $J^{n+1}=I J^{n}$ implies $v_{i}(J)=v_{i}(I)$ for all $i$. Upon setting $d_{i}=$ $\left[T^{\prime} / M_{i}: T / M T\right]$ we have $e(J)=\sum d_{i} v_{i}(J)$.

REMARK. Corollary 1.2 extends to all local rings as Theorem 2.3 in [5] extends to Theorem 3.2. This is accomplished by passing to the completion and using the associativity formula to reduce to the case where $R$ is a complete local domain, hence analytically unramified.

Before proceeding to the second theorem of Rees we recall the notion of integral dependence on an ideal. An element $x$ in $R$ is said to be integral over an ideal $I$ if $x$ satisfies an equation of the form

$$
x^{n}+i_{1} x^{n-1}+\cdots+i_{n}=0, \quad i_{k} \in I^{k}, 1 \leq k \leq n .
$$

The set of elements $\bar{I}$ integral over $I$ form an ideal and for any ideal $J \supseteq I, I$ reduces $J$ if and only if $\bar{I}=\bar{J}$. 
COROLlaRY 1.3 ([6], THEOREM 3.2). Let $(R, M)$ be a quasi-unmixed local ring and $I \subseteq J M$-primary ideals. If $e(I)=e(J)$ then $\bar{I}=\bar{J}$.

PROOF. Recall that $R$ is quasi-unmixed in case its completion is equidimensional. Then as in [6], one uses the associativity formula to reduce to the case where $R$ is a complete local domain (this is the only place where the quasi-unmixed hypothesis is required). Since $R / M$ is infinite, we may replace $I$ by a minimal reduction generated by a system of parameters, say $x_{1}, \ldots, x_{d}$. Changing notation so that $I=\left(x_{1}, \ldots, x_{d}\right) R$ and with $T$ as in the theorem we have $e(J)=e(I)=e(I T) \geq$ $e(J T)$. To see $e(J T) \geq e(J)$ write $T=S / K$ where $S=R\left(Z_{1}, \ldots, Z_{d-1}\right)$ and set $L=\left(x_{d} Z_{1}-x_{1}, \ldots, x_{d} Z_{d-1}-x_{d-1}\right) S$. Since $L$ is part of a system of parameters for $S, e(J)=e(J S) \leq e(J S / L)$. As before, $\operatorname{dim} K / L<\operatorname{dim} S / L$ (as $S / L$-modules) so $e(J S / L)=e(J T)$. Thus $e(I T)=e(J T)$. In the notation of Corollary 1.2,

$$
e(I T)=\sum_{i} d_{i} v_{i}(I) \quad \text { and } \quad e(J T)=\sum_{i} d_{i} v_{i}(J)
$$

Since $v_{i}(J) \leq v_{i}(I)$ and $d_{i}>0$ for all $i$, we must have $v_{i}(J)=v_{i}(I)$ for all $i$. Thus

$$
J \subseteq \bigcap_{i} I T_{M_{i}}^{\prime} \cap R=\bar{I}
$$

(say, by [1] , Proposition 3). Thus $\bar{I}=\bar{J}$ and the proof is complete.

2. Teissier's theorem. In [9 and 10] Teissier proved the following theorem.

THEOREM. Let $(R, M)$ be a d-dimensional Cohen-Macaulay complex analytic algebra and $I, J \subseteq R, M$-primary ideals. Then

(1) $e(I J)^{1 / d} \leq e(I)^{1 / d}+e(J)^{1 / d}$.

(2) Equality holds in (1) if and only if there exist positive integers a and b satisfying

$$
\overline{I^{a}}=\overline{J^{b}} \text {. }
$$

Teissier's proof contains two essential algebraic ingredients. The first is to involve the so-called mixed multiplicities of $I$ and $J$ and the second is to provide a reduction procedure (really a strengthened superficial element argument) whereby the theorem can be reduced to the two dimensional case. In [7] Rees and Sharp give a purely algebraic proof of Teissier's theorem showing that (1) holds for any two dimensional local ring and (2) holds for any two dimensional quasi-unmixed local ring. They observe that Teissier's reduction carries over for (1) so that the inequality remains valid for any local ring. The apparent obstruction to this procedure as it applies to part (2) of the theorem is overcome by adopting the view put forth in this note. We shall indicate the required reduction as painlessly as possible (by assuming familiarity with $[\mathbf{8}, 9$ and 10]).

PROPOSITION 2.1. Part (2) of Teissier's theorem holds for any quasi-unmixed local ring of dimension $d \geq 2$.

PROOF. Let $e_{0}=e_{0}(I / J), \ldots, e_{d}=e_{d}(I / J)$ denote the mixed multiplicities of $I$ and $J$ defined in [7]. As Teissier shows in [10], the proposition reduces to proving the following assertion. If $e_{0}=\cdots=e_{d}$, then $\bar{I}=\bar{J}$. The proof now proceeds by induction on $d$, the case $d=2$ being proved in [7]. We indicate how the result follows from the $d-1$ case in several steps. 
(a) We may replace $I$ by a minimal reduction and assume that it is generated by a system of parameters, say $x_{1}, \ldots, x_{d}$. Neither the mixed multiplicities nor the integral closures are disturbed.

(b) It follows from [8], Proposition 2.1, that there exist $r_{0} \geq 0$ and $y_{1} \in I$ such that

$$
\left(I^{r} J^{s}: y_{1}\right) \cap I^{r_{0}} J^{s}=I^{r-1} J^{s}
$$

for all $r>r_{0}$ and $s \geq 0$. It can be shown that $y_{1}$ may be chosen to satisfy $I=\left(y_{1}, x_{2}, \ldots, x_{d}\right)$.

(c) For an indeterminate $Z$, set $g=x_{2} Z-y_{1}$. Then it is readily seen (as in the proof of Theorem 1.1) that

$$
\left(I^{r} J^{s} R(Z): g\right) \cap I^{r_{0}} J^{s} R(Z)=I^{r-1} J^{s} R(Z)
$$

for all $r>r_{0}$ and $s \geq 0$.

(d) As in the proof of [8], Proposition 2.1, this implies that

$$
e_{0}=e_{0}^{\prime}, \ldots, e_{d-1}=e_{d-1}^{\prime}
$$

where $e_{0}^{\prime}, \ldots, e_{d-1}^{\prime}$ are the mixed multiplicities of $I$ and $J$ as ideals of $R(Z) /(g)$.

(e) $R(Z) /(g)$ is quasi-unmixed and has dimension $d-1$. Therefore by induction $J R(Z) /(g) \subseteq \overline{I R(Z) /(g)}$.

(f) Let $P \subseteq R$ be any minimal prime, write $\tilde{R}$ for $R / P$ and set

$$
T=\tilde{R}\left[y_{1} / x_{2}\right]_{M \tilde{R}\left[y_{1} / x_{2}\right]} .
$$

Since $R(Z) /(g)$ maps onto $T, J T \subset \overline{I T}$ and because $\tilde{R}$ is quasi-unmixed, $\overline{I T} \cap \tilde{R}=$ $\overline{I \tilde{R}}$ (as in the proof of [1], Proposition 3). Thus $J \tilde{R} \subseteq \overline{I \tilde{R}}$.

Since this holds for each minimal prime, $J \subseteq \bar{I}$.

(g) By symmetry, $I \subseteq \bar{J}$, and the proof is complete.

ACKNOWLEDGEMENT. I would like to thank Professor Jugal Verma for many enlightening conversations concerning the topics in this paper and the referee for pointing out places where additional clarification was required.

\section{REFERENCES}

1. D. Katz, On the number of minimal prime ideals in the completion of a local domain, Rocky Mountain J. Math. 16 (1986), 575-578.

2. D. G. Northcott, Lessons on rings, modules and multiplicities, Cambridge Univ. Press, Cambridge, 1968.

3. D. G. Northcott and D. Rees, Reductions of ideals in local rings, Math. Proc. Cambridge Philos. Soc. 50 (1954), 145-158.

4. D. Rees, A note on analytically unramified local rings, J. London Math. Soc. 36 (1961), 24-28.

5. _ Degree functions in local rings, Math. Proc. Cambridge Philos. Soc. 57 (1961), 1-7.

6. $\_, \mathscr{A}$-transforms of local rings and a theorem on multiplicities of ideals, Math. Proc. Cambridge Philos. Soc. 57 (1961), 8-17.

7. D. Rees and R. Y. Sharp, On a theorem of B. Teissier on multiplicities of ideals in local rings, J. London Math. Soc. 18 (1978), 449-463.

8. B. Teissier, Cycles evanescents, sections planes, et conditions de Whitney, Singularités à Cargése 1972, Astérique 7-8 (1973).

9. __ Sur une inégalité à la Minkowski pour les multiplicités (Appendix to a paper by D. Eisenbud and H. I. Levine), Ann. of Math. (2) 106 (1977), 38-44. 
10. ___ On a Minkowski-type inequality for multiplicities-II, C. P. Ramanujam-A Tribute, Tata Institute Studies in Mathematics, Springer-Verlag, Berlin and New York, 1978, pp. 347-361.

11. O. Zariski and P. Samuel, Commutative algebra, vol. II, Van Nostrand Reinhold, New York, N. Y., 1960.

DEPARTMENT OF MATHEMATICS, UNIVERSity OF KANSAS, LAWRENCE, KANSAS 66045 